\title{
Économie et pouvoir au sein du parc national des
} Écrins

Penser la nature, définir l'espace

\section{Valeria Siniscalchi}

\section{(2) OpenEdition \\ Journals}

Édition électronique

URL : https://journals.openedition.org/tc/3941

DOI : 10.4000/tc.3941

ISSN : 1952-420X

\section{Éditeur}

Éditions de l'EHESS

Édition imprimée

Date de publication : 31 décembre 2008

Pagination : 40-59

ISSN : 0248-6016

Référence électronique

Valeria Siniscalchi, «Économie et pouvoir au sein du parc national des Écrins », Techniques \& Culture [En ligne], 50 | 2008, mis en ligne le 31 décembre 2010, consulté le 29 septembre 2022. URL : http:// journals.openedition.org/tc/3941; DOI : https://doi.org/10.4000/tc.3941 


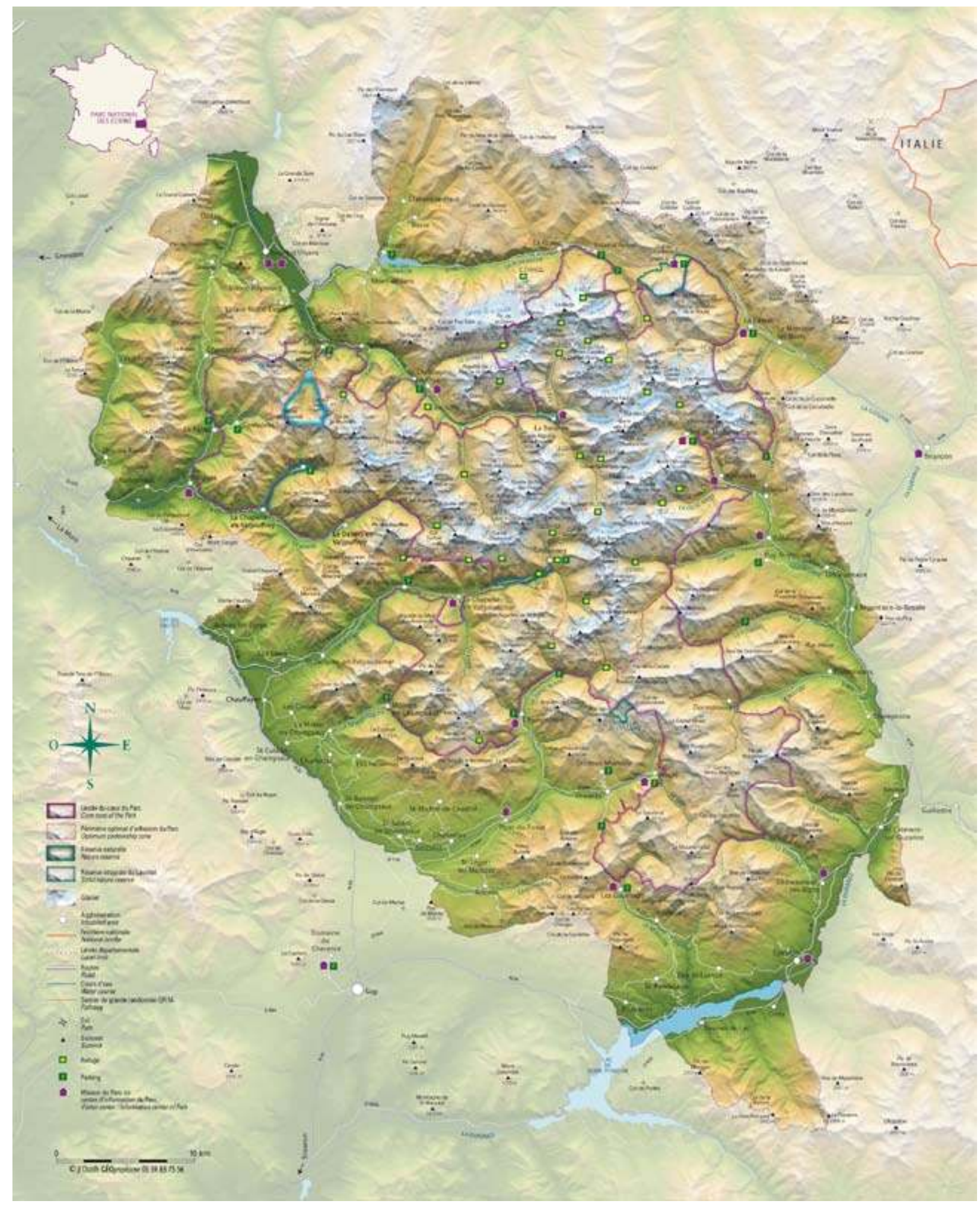




\title{
ÉCONOMIE ET POUVOIR
}

\section{AU SEIN DU PARC NATIONAL DES ÉCRINS}

\section{Penser la nature, définir l'espace}

\author{
Cet article sur les Écrins retrace les processus de patrimonialisation de la nature \\ et de définition de l'espace protégé, en déployant les logiques économiques et \\ les enjeux politiques qui les accompagnent.
}

Les espaces protégés sont des lieux d'observation privilégiés, au sein desquels s'expriment des logiques d'utilisation du territoire et des intérêts divergents et à travers lesquels les conflits se révèlent souvent. En suivant les transformations des actions et des discours du Parc national des Écrins au cours des dernières décennies, il s'agit ici de comprendre et démêler une partie des luttes symboliques et réelles qui caractérisent son histoire. Lanalyse se concentre sur les modifications de l'idée de nature et la qualification de cette dernière en termes patrimoniaux. Aux transformations des notions utilisées par le parc correspondent des changements de posture et d'actions, qui sont à leur tour révélateurs des changements survenus au niveau des enjeux politiques et économiques impliquant le parc. J'appréhende par conséquent le parc comme l'un des acteurs politiques de la scène locale et supra-locale, plus que comme une institution représentante de l'État et agissant dans l'intérêt commun. Cet article examine les politiques et les rhétoriques à travers lesquelles cette institution est devenue l'une des protagonistes du processus de construction de l'espace. Les analyses présentées ici font partie d'un travail ethnographique plus large qui porte sur la construction et la 
définition des lieux et sur les enjeux de la gestion du territoire, mené dans le département des Hautes-Alpes entre 2000 et 2004'.

La démarche adoptée considère les rhétoriques et les discours sur le territoire produits par le parc comme ayant une valeur performative. Les actes linguistiques ne sont pas seulement des «manières de dire »; ce sont aussi des "manières de faire » la réalité sociale, des modes d'affrontement, de négociation et de production du sens (cf. Certeau 1990, Herzfeld 1997). Lanalyse sémantique que je propose des documents officiels du parc, de ses politiques et des visions de ses agents, s'accompagne de l'analyse des pratiques, considérées elles aussi dans leurs dimensions expressive et performative.

\section{La nature contre léconomie}

Le Parc national des Écrins - le cinquième des neuf parcs nationaux français - a été institué par décret (D73-378 du 27 mars 1973) sur un territoire où s'étaient succédé depuis le début du XXe siècle diverses formes anticipatrices de protection de la nature. Le "parc de la Bérarde », mis en place en 1913 autour de la haute vallée du Vénéon, était devenu par la suite « Parc national de l'Oisans », puis, en 1924, « Parc national du Pelvoux ». Lorigine du parc de la Bérarde remonte à l'achat par l'État de pâturages situés sur la commune de Saint Christophe en Oisans. Le but de l'acquisition n'était pas de reboiser, contrairement à la pratique mise en œuvre à cette époque pour faire face aux dégâts dus au surpâturage (érosion, inondations, etc.). Lidée qui présidait à cette intervention de l'État était celle de la création d'un parc afin de soustraire ces espaces à l'action des hommes - y compris des forestiers -, en laissant la nature faire elle-même son travail de restauration.

Une fois le parc créé, le territoire protégé s'élargit progressivement: du Vénéon à la Vallouise puis au Valgaudemar. Le « parc de la Bérarde » devient alors « Parc national du Pelvoux».

Ces premières formes du parc ont été élaborées à un moment particulier de l'histoire des rapports à la montagne et de l'histoire des formes de protection de l'espace très différentes de celles que l'on peut observer aujourd'hui. D'après la reconstruction historique qu'en fait Zuanon (1995 : 20), le problème de la dégradation des terrains de montagne, le développement de l'alpinisme et un sentiment écologiste ante litteram animé surtout par « des considérations d'ordre esthétique » sont à la base des trois courants de pensée, parfois convergents, qui conduisent au renforcement de l'idée de protection de la montagne. Les thèmes qui fédèrent partiellement les sociétés alpines et les forestiers sont ceux de l'appropriation matérielle et symbolique de l'espace montagnard et de l'intervention « civilisatrice» auprès de ses habitants (ibid. : 36). On trouve d'ailleurs à l'origine du projet de parc un haut fonctionnaire des 
Eaux et Forêts, Alphonse Mathey. Ce dernier a une vision non-interventionniste, contrairement à une grande partie de ses collègues forestiers (ibid. : 69-78). Le cadre de référence dans lequel son projet mûrit est celui d'une nature abîmée par l'homme et devant être protégée afin de pouvoir rétablir elle-même ses équilibres internes. Ces éléments historiques permettent d'éclairer les oppositions qui précéderont la constitution d'un « vrai » parc national et les conflits vis-à-vis de l'institution qui s'expriment depuis sa naissance, même si la vente à l'État des terrains qui ont constitué le premier noyau du parc s'est passée sans trop de problèmes - d'après les documents historiques utilisés par Zuanon - car elle constituait une source de revenus pour les communes.

Deux ans après la loi de création des parcs nationaux, en 1962, le parc perd sa qualification de parc national et devient un parc domanial, « domaine privé de l'État soumis au régime forestier » (Avocat 1977 : 2-4); mais, dans les années qui suivent la loi de 1960, les réflexions autour de la possibilité de créer un « vrai » parc national dans le massif du Pelvoux s'intensifient.

Dans les deux départements sur lesquels s'étend aujourd'hui le parc des Écrins - les Hautes-Alpes et l'Isère - une bonne partie des élus est alors opposée au projet. Il est facile de comprendre les raisons de cette résistance.

« Les oppositions fortes qu'il y a eues et qui de fait existent encore sont des oppositions entre l'État et des contextes locaux. Ici déjà, il y avait eu le traumatisme avec le RTM [Restauration des terrains en Montagne] à la fin du XIX ${ }^{e}$ siècle: l'État a exproprié des villages entiers pour y installer des périmètres à reboiser. Cela a été un exemple de la politique dure imposée par l'État. À cette époque, il avait besoin de s'imposer, il l'a fait avec des moyens forts qui ont traumatisé les gens [...]. La venue du parc a été mal vécue. Entre 1965 et 1970, il y a vraiment eu une mauvaise communication, on disait aux gens qu'ils ne pouvaient plus rien faire » (H.H. chef de secteur).

Si le parc de la Bérarde d'abord et le parc du Pelvoux ensuite avaient été institués dans des terrains désormais sans valeur économique, la mémoire des acquisitions de la part de l'État avait probablement laissé des traces. En outre, les perspectives économiques des années 1960 ne sont plus celles du début du XX $\mathrm{X}^{\mathrm{e}}$ siècle. Les sports de nature commencent à se développer et les Alpes apparaissent comme un terrain particulièrement favorable à ce genre d'activités. Du point de vue de la plupart des communes, le parc viendrait soustraire des espaces au développement économique, lequel s'exprime à cette époque par l'implantation de stations de ski. Cette position se renforcera surtout après la création du parc actuel. A contrario, du point de vue des porteurs du projet, ces mêmes arguments justifient l'intérêt de la création d'un parc national. Les arguments utilisés en faveur de ce dernier mettent l'accent sur la richesse de la flore et de la faune, sur l'idée d'un équilibre naturel à préserver et sur la richesse du paysage. Ils procèdent de l'esprit qui avait guidé la création du parc de la Bérarde, puis du Pelvoux; ils se situent également dans le prolongement direct des critères qui, selon la loi 60-708 du 22 juillet 1960, permettent de classer un territoire en « parc national» : 

« Les limites ne sont pas toujours ce qu'elles devraient être au strict plan de la protection des richesses naturelles; en particulier, il faut bien reconnaître une grande part d'artifice dans la délimitation même du parc et de la zone périphérique, mais peut-il en être autrement? Trop d'intérêts sont en jeu pour que les exigences scientifiques puissent être intégralement satisfaites », écrit Charles Avocat - géographe et membre du Comité scientifique du parc - trois ans après sa création (Avocat 1977 : 11-12).

Même si le décret qui institue un parc national fixe le périmètre de sa zone centrale qui ne sera plus modifié, la question de la définition des limites est récurrente tout au long de la vie du Parc national des Écrins: elle apparaît dès le début de son existence et, même si elle est exprimée différemment au fil des époques, elle reste un enjeu dans les politiques actuelles du parc, y compris jusqu'aux modifications législatives plus récentes².

Parmi les acteurs qui ont joué un rôle dans la définition initiale du périmètre du parc, les sociétés de chasse ont occupé une place importante. Comme le souligne entre autres Joseph Szarka, dans son analyse de la politique environnementale française, le pouvoir du lobby des chasseurs est capable, aujourd'hui encore, de bloquer en partie la législation européenne concernant la conservation et la protection de la nature (2002 : 135-138).

En dépit des négociations et des efforts qui semblent avoir été faits pour prendre en compte les exigences des différents groupes de pouvoir dans les territoires concernés par le parc, surtout dans les premières années, les relations du parc avec les chasseurs et les communes à propos de la réglementation de la chasse restent tendues et donnent lieu à des conflits permanents. Plus généralement, à cette période, les discours de l'institution et ceux des acteurs locaux apparaissent radicalement opposés:

« Le premier directeur du parc était très « nature », il était pour ainsi dire contre les habitants et, selon lui, la zone périphérique n'existait pas. C'était un militant écolo. Il était contre les chasseurs qui, eux, étaient puissants et il y a presque eu la guerre avec lui » (B.B. chargé de mission).

À l'époque, les objectifs prioritaires du parc sont de préserver des espaces, de les observer d'un point de vue scientifique, de faire découvrir la montagne aux visiteurs et de respecter les témoignages de la présence humaine. Dans la pratique, les actions de son personnel semblent essentiellement tournées vers la préservation d'une « nature » faite de flore, faune, sols et eaux, et vers sa protection contre l'action destructrice de l'homme, au cœur d'un espace qui, au cours des années 1970, tend à être perçu comme un "sanctuaire », comme le définissent quelques-uns des responsables du parc qui analysent a posteriori cette période. Il s'agit d'une posture proche de celle qui, soixante ans auparavant, avait caractérisé la mise en place du parc de la Bérarde. Le souci de «préservation » semble opposer le parc, encore plus fortement que dans les années qui précèdent sa création, au développement touristique du département et des communes situées dans la zone centrale. En revanche, en zone périphérique, dans le Champsaur, dans le Briançonnais et dans l'Embrunais, les stations de ski continuent à se développer. 


\section{Le patrimoine bâti et les paysages}

Ils deviennent les signes de laction de l'homme et à la fois les nouveaux outils conceptuels des actions du parc (L'Écho des Écrins. Le journal d'information du Parc Nationa/ 14, automne 2000).

(Fig. 2)
La tension entre conservation de la nature et développement économique apparaît également récurrente dans les autres parcs nationaux (cf. Szarka 2002 : 127). Même s'il serait réducteur de lire les conflits entre l'institution et le contexte local uniquement en ces termes.

Cette apparente dichotomie des positions ne doit pas non plus conduire à adopter une vision dichotomique de la réalité observée. Je ne considère pas le parc comme une institution qui agit en fédérant des intérêts particuliers au nom de la protection de l'environnement, et en s'opposant ou se confrontant à des « populations locales", ces dernières « imaginées » (cf. Anderson 1991) comme un ensemble compact, doté d'intérêts homogènes et nettement différencié de l'institution parc. Comme j'ai pu déjà le souligner ailleurs (Siniscalchi 2007), les gestionnaires du parc ne constituent pas non plus un ensemble homogène séparé du contexte local. Cette vision simplificatrice, répandue dans le discours commun tout comme dans une partie de la littérature scientifique, crée et essentialise des entités qui ne correspondent pas à la complexité des contextes réels, dans lesquels les acteurs - les agents du parc tout comme les élus ou les habitants des communes au sein du parc - assument des positions et des rôles multiples. En outre, le discours officiel du parc, sa politique et les actions de ses agents, ainsi que les intérêts des uns et des autres, changent dans le temps.

\section{Le patrimoine pour l'économie}

Des années 1960 à nos jours, des transformations importantes se sont produites dans les formes de protection de la nature. Le passage de l'idée d'une nature à préserver à la notion de « patrimoine naturel » d'abord, et « culturel » ensuite, a accompagné ce processus. Parallèlement, ce changement est révélateur des modifications, à certains égards radicales, qui 


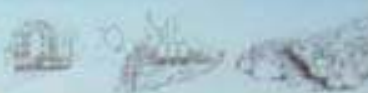

des metiers pour les restaurer ef les entretenir

\section{construit}
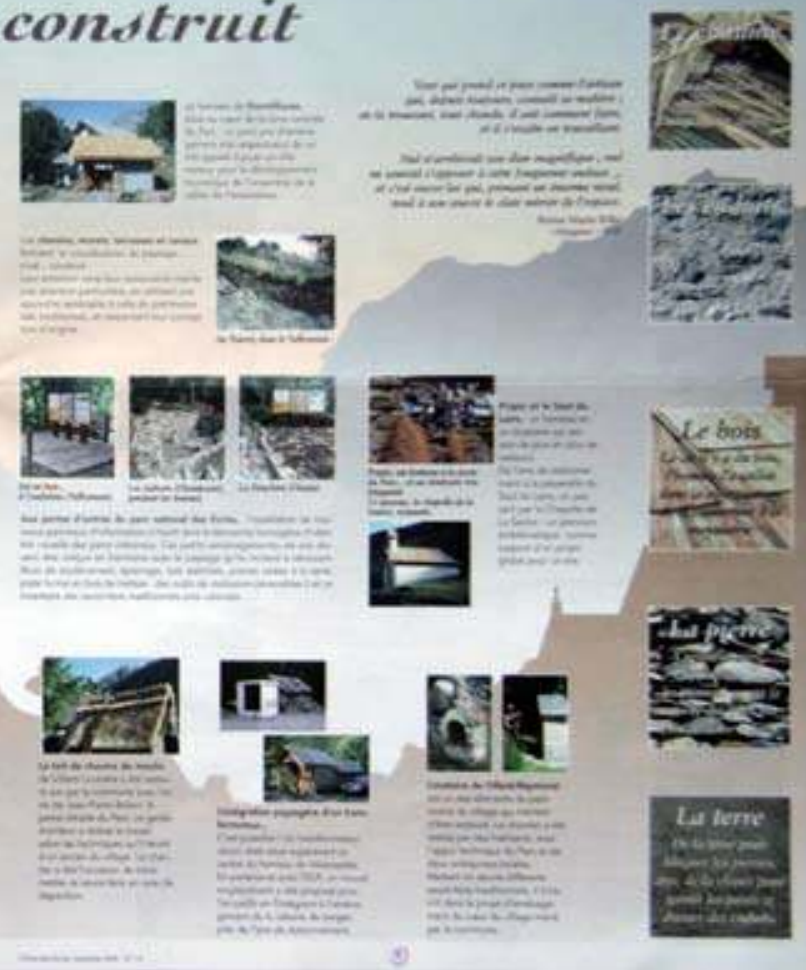

s)
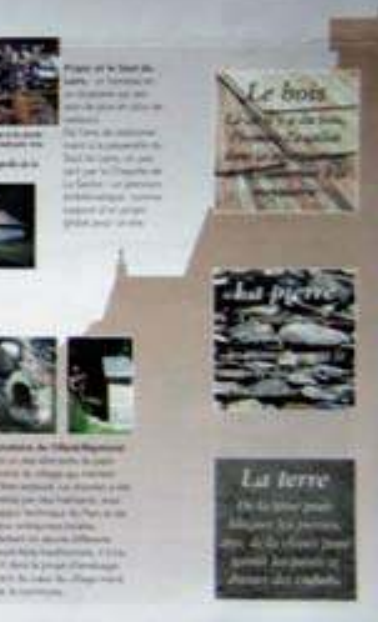

ont eu lieu dans les rapports entre le parc en tant qu'institution et le contexte local. Le nouveau directeur nommé au début des années 1990 vient du parc des Cévennes, l'un des deux parcs nationaux (avec le parc de PortCros) dont la zone centrale est habitée. Sous sa direction, en l'espace de dix ans, des changements majeurs se sont produits. Ils concernent tout d'abord la politique et le discours du parc, ensuite ses actions en zone périphérique, jusque-là plutôt considérée comme une zone de compensation, dans laquelle le parc n'était pas de fait présent sinon à travers quelques financements disparates. Le « patrimoine » devient un outil, d'abord conceptuel, pour permettre au parc d'intervenir dans l'ensemble de son territoire, y compris la zone périphérique.

Lhistoire de la notion de " patrimoine » est complexe et la littérature sur ce thème est désormais très vaste. Ce qu'il importe de souligner ici est qu'au fil du temps le sens du mot « patrimoine » s'est élargi, et que ce phénomène sémantique s'est accompagné d'une diversification des pratiques de protection et de conservation. Le « patrimoine » est ainsi devenu à la fois un enjeu politique et un objet d'intervention et de recherche. Le patrimoine existe quand un pouvoir politique le rend légitime. Dans cette perspective, je ne considère pas le patrimoine comme un donné ou un instrument d'analyse, mais au contraire comme un objet à déconstruire, une notion utilisée et appropriée par les différents acteurs et dont le sens et les usages changent en permanence.

Selon Lefeuvre, en France le terme « patrimoine naturel » est apparu pour la première fois au moment de l'institution des parcs naturels régionaux, dans un document officiel qui affirme: « un territoire peut être classé dans cette catégorie en raison de "la qualité de son patrimoine naturel et culturel" » (1990: 29). Desvallées, en retraçant l'histoire du mot «patrimoine » et de son utilisation dans la tradition institutionnelle française, estime au contraire que l'usage qui en est fait à partir de la fin des années 1960 est plutôt une résurgence: en 1956, déjà, le terme avait été employé 


\section{La vallée du Fournel: comment gérer ensemble un espace naturel}

Comment proteger le vallon

preserver son patrimoine naturel et y rèaliser

les différents amenagements qui peuvent savèrer nécessaires ? La réponse viendra de la concertation.

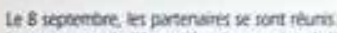
lo commune de rNyentiere fon mare, dot

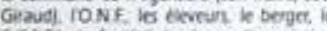

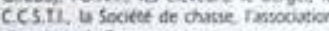

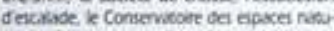
nets de hovence et $x$ farc in preboble une approche descrgeve os miles rtaite par

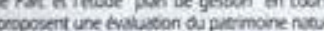
red Chique parterave ayant ies propes prio. fete be returion de trivel dot permetire de

defini les objecth de getion et de progran

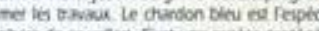

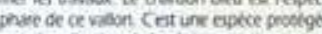
mis rappication de la ritgementation ne iu

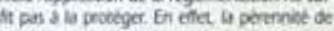
th Reine de Aiperi. est do a raction de momer une fuchrison tad in stoon pet. met une bonre forason et ty pegineeston de ge phrite undis ge freindon of cete pr?

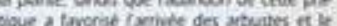
phurage sur lea statons de chardon beur en

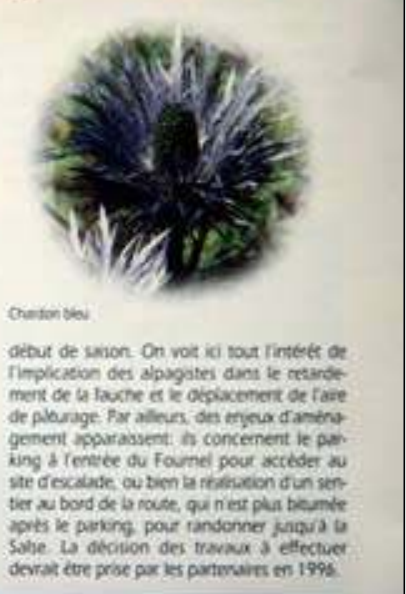

\section{L'espace naturel devient} un élément patrimonial

(L'Écho des Écrins. Le journal d'information du Parc Nationa/2, novembre 1995).

(Fig. 3) dans un article où l'on parlait de « patrimoine culturel, archéologique, historique, artistique et naturel de la France $»^{3}$ (1998: 93). Selon Audrerie, c'est en 1971 « avec la nomination de Robert Poujade comme ministre délégué auprès du Premier ministre, chargé de la Protection de la Nature et de l'Environnement » qu'en France la notion de patrimoine s'élargit à l'environnement dans son ensemble (1997 : 24). La création, au début des années 1970, des départements ministériels de l'Environnement, « est un symptôme de la reconnaissance de l'environnement comme problème social et politique » (Kalaora 1997 : 182). Mais, selon Lefeuvre, en 1980 (pourtant déclarée « année du patrimoine »), « l'État et les administrations centrales [...], l'envisagent encore uniquement sous les aspects architecturaux et culturels, et ceci malgré plusieurs interventions des associations de protection de la nature » $(1990: 47)$. Si l'on se réfère aux interventions institutionnelles dans ce domaine, le ministère de l'Environnement semble surtout avoir pris en compte une « nature exemplaire plutôt que la nature ordinaire » (Kalaora 1997 :185), afin de distinguer les actions de ce ministère par rapport à celles du ministère de l'Agriculture.

Sur le plan international, l'Unesco adopte en 1972 une « Convention pour la protection du patrimoine mondial culturel et naturel $\gg$ (Chastel 1997 : 1463). Pour Lefeuvre, il s'agit là d'un signal: « la séparation entre homme et nature, entre sciences naturalistes et sciences humaines va s'atténuer au cours de la décennie 1970-1980» (1990: 40).

Le « patrimoine culturel », déjà légitime, permet un élargissement de la notion de patrimoine à la nature et à l'environnement. De manière symétrique, la protection des milieux écologiques incite à prendre en compte le suivi et la gestion au quotidien de ce que l'on considère comme du patrimoine, en le reconnaissant dans ses caractéristiques vivantes et comme étant en évolution permanente (Audrerie 1997 : 88).

Dans la loi de Protection de la nature de 1976, le terme «patrimoine naturel » n'apparaît plus associé à celui de « patrimoine culturel » (Lefeuvre 1990 : 29), et de plus en plus il sera considéré indépendamment de la 
dimension culturelle. « L'ensemble législatif français [... ] mettra du temps à se démarquer d'une conception trop naturaliste de la protection de la nature » (ibid. : 37), à l'origine de cette séparation entre homme et nature.

Dans les textes ou les documents du Parc national des Écrins, le terme de «patrimoine » n'apparaît que tardivement et, lorsque c'est le cas, son acception est d'abord celle de «patrimoine naturel ». Dans le « Programme d'aménagement » pour les années 1991-1995², par exemple, le « patrimoine naturel » et le paysage, déclinés en patrimoines floristique, faunistique et géologique, occupent le premier chapitre du volume. Seules trois pages, vers la fin du texte, sont dédiées au patrimoine culturel. Dans le numéro 2 de L'Écho des Écrins, daté de novembre 1995, un dossier concerne le "patrimoine »: on y trouve deux articles «Comment gérer ensemble un espace naturel » (Fig. 3) et « Le bâti rural: empreintes et lieux de vie ». Dans le premier, le terme de patrimoine est utilisé au sens de patrimoine naturel, dans le second il s'agit de patrimoine bâti. À la fin de ce même numéro, un petit article concernant des opérations d'aménagements architecturaux aborde la question du « savoir-faire traditionnel ». Dans le numéro 4 de janvier 1997, le même Écho des Écrins dédie son dossier central au « patrimoine naturel ». Quand l'économie est prise en compte, dans le discours et dans les pratiques du parc, le thème du développement économique passe par celui de la protection du patrimoine naturel. Mais l'association entre « patrimoine naturel » et développement économique ne semble pas encore en mesure de faire dialoguer le parc et les communes. La notion de patrimoine ne semble pas non plus modifier radicalement l'idée, à la base de la création d'un parc national, d'une nature qualifiée d'exceptionnelle et à préserver:

« L'existence d'un espace naturel aussi exceptionnel que celui des Écrins est un atout pour l'économie de ses vallées. La création d'un parc national signe sa reconnaissance par l'État. Ainsi ce patrimoine préservé prend une valeur supplémentaire... particulièrement profitable à l'activité touristique » (L'Écho des Écrins, janvier mars 1998: 4).

Néanmoins, la nouvelle vision patrimoniale permet au parc de se présenter comme l'un des acteurs du développement et de réintroduire, parmi ses intérêts et d'une manière explicite, la dimension économique.

Quelques modifications se produisent lorsque l'accent est mis sur les paysages et lorsque, par ce biais, le parc commence à prendre en compte les savoir-faire. Ce processus est décelable dans certaines des actions que le parc accompagne ou encourage en zone périphérique, comme celles qui concernent les savoir-faire du bocage. Le bocage du Champsaur est l'un des paysages présenté comme exemple, dans le dossier central du numéro 3 de L'Écho des Écrins (mars 1996), « Espaces des hommes ». « Le maintien d'un

Le parc et léconomie locale À travers le patrimoine, le parc est présenté comme l'un des acteurs du développement économique du territoire (L'Écho des Écrins. Le journal d'information du Parc Nationa/8, hiver 1998).

(Fig. 4) 
paysage bocager équilibré répond à plusieurs objectifs complémentaires, concernant l'agriculture, la nature [...] et le tourisme » affirme l'article. Dans ce texte, le terme « patrimoine » n'apparaît pas. Un deuxième article sur le bocage de la vallée du Champsaur est publié en 1999 (dans le numéro 10 de L'Écho des Écrins). Cette fois, les mots ont changé: « Le bocage est un patrimoine qui mérite d'être conservé. Et pour maintenir ce paysage, on ne peut plus « culturel », il est nécessaire de lui (re)donner sa valeur économique ( (Fig. 5). Le bocage ne constitue pas simplement un paysage, mais en tant que tel il devient un élément du patrimoine «culturel». La vision de plus en plus patrimoniale de l'espace permet une prise en compte simultanée des aspects naturels et humains.

\section{Le Champsaur, jardin alpin du pays bocager}

C'cot une vallice de mootagne jus condinaite ron la

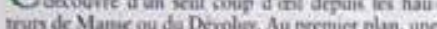

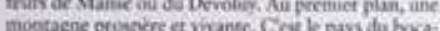
ge, aves ses recear de haies, sos canase, se de ge, avec ses reserux de haies, so canaux, so de mins creva et ses arkice tritanks, comme is Saint.

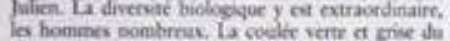

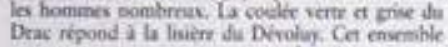
compose un pysage monimgnand, voire campacnand, a contemgiler depuis be hel védere des Troes Croix ou des Riscris. Cette vie sujound hui dispence an hancass, a lecart de gros bourp comine

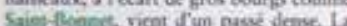

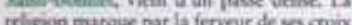

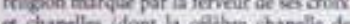
et chapelles dont la chichve chaprite da Petien

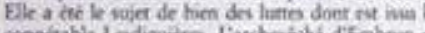
connctable lodiguitro. Laechrciche d'Emitran son pouvoir a vocicde a une noblesse de tertoit né

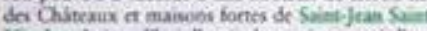
Sigolas. Aljourd hai, Fagricaltore vient aussi d une

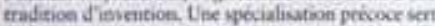
de hace a un tourisme vat equ s'appuie sur le quas.

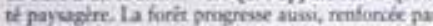

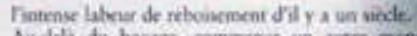
Ausela du bocage, commence un satre monde, cdui ic $\mathrm{b}$ havie montage. Thoenene en prises maik crte occuputive trahit des laiblesses isvoo $\&$

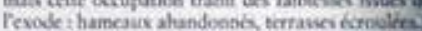
Une tradition aero-pastorale se maineicnt sutoar de stations de sponts dhiver. Leté, L plupart des val loni et alpages sonit stilines par los ovina et lo

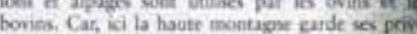

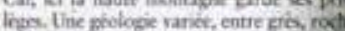
nolcaniquar, yranitc ef thysh, es lacectef polos Dras a curs afflucest. Frotige of py

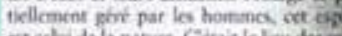

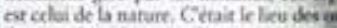
it pose les chamois, les marmoters.

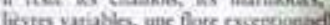

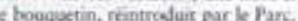

Cert un espace d'aventure poar ks randenenes a les alpinises quir ratoicnt Thahinde da cosur do man

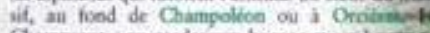
Champuser a su garder soi bocage et ser houninecisu wane ervogant ses ramifications a lassgis

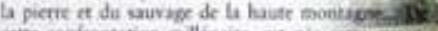
ponecile valles.

\section{Le paysage devient patrimoine culturel}

Le bocage du Champsaur. (L'Écho des Écrins. Le journal d'information du Parc Nationa/ 10, été 1999).

(Fig. 5)
C'est dans une vidéocassette qui traite de ce même thème - « Derrière la haie $»$ - produite par le parc en décembre 2001, dans le cadre de l'un des projets financés par le programme européen Leader II, que le changement est le plus visible (Fig. 7). Après une illustration des techniques utilisées et des savoir-faire nécessaires à l'entretien des haies qui dessinent et construisent le paysage, puis de la fonction du bocage dans l'économie agricole et de sa valeur patrimoniale, un représentant du parc conclut en ces termes:

« Notre rôle c'est de conserver un héritage pour des générations futures [...] autrefois on transmettait son bien, aujourd'hui on va transmettre un ensemble de valeurs et ces valeurs, elles transparaissent dans des paysages. Qu'est-ce qu'on est capable de laisser à nos successeurs? » (H.H. chef de secteur). 
Après la projection de ce film, lors du vernissage d'une exposition sur le bocage, ce même agent m'expliquait:

« Je crois qu'en définitive les protections culturelle et naturelle vont ensemble. Par exemple avec le bocage, ce qu'il faut conserver c'est un paysage, mais surtout un savoir-faire » (H.H. chef de secteur).

Dans le même esprit, à l'automne 2000, le dossier central de L'Écho des Écrins 14 est dédié aux savoir-faire, au patrimoine bâti et au paysage:

« Les espaces naturels protégés n'ignorent plus l'histoire culturelle de leur territoire. Habitat, paysage, savoir faire et techniques traditionnelles constituent un héritage indispensable pour gérer au quotidien ces patrimoines. »

Ces exemples sont les signes de changements plus larges en train de se produire au sein du Parc national des Écrins. Le parc et son personnel sont de plus en plus attentifs aux techniques et aux savoirs à travers lesquels le paysage et plus en général le territoire ont été modelés: des techniques et des savoirs qui assument désormais le rôle de «valeurs ». Et le milieu naturel n'est plus un ensemble de flore, faune, sols et eaux desquels l'action humaine est exclue. La « nature » se transforme sous l'action de l'homme, et c'est la « culture » qui devient garante de continuité.

« Le ministère de l'Environnement nous confie le parc naturel, sa sauvegarde et sa gestion, ce qui signifie maintien des milieux et des habitats; or milieux et habitats veulent dire aussi usages et pratiques, il faut donc également gérer le patrimoine culturel $»$ (F.F. chef de secteur).

Pour comprendre ces changements, il faut les situer dans le contexte plus ample dans lequel s'inscrit le parc, et pour cela faire référence aux politiques des ministères dont il est l'émanation ou avec lesquels il entretient des relations. Entre 1979 et 1988, les interactions entre la direction du Patrimoine et les parcs naturels (régionaux et nationaux), sous des formes diverses, avaient contribué à stimuler l'intérêt à l'égard des savoirs, des savoir-faire et des connaissances des habitants des espaces protégés. Au milieu des années 1990, on (re)commence aussi à parler de patrimoine culturel dans les parcs nationaux ${ }^{5}$.

"La conservation du patrimoine naturel [...] ne peut se satisfaire du "gel" pur et simple de l'espace parce que l'activité humaine a souvent contribué à façonner les valeurs que nous estimons aujourd'hui [...]. L'approche des parcs nationaux reste essentiellement patrimoniale. Elle intègre donc les dimensions culturelle et identitaire des territoires. $»^{6}$

Si une partie de ces transformations est donc liée à des changements de perspective au niveau politique national, le processus en cours dans le Parc national des Écrins présente des spécificités et on a l'impression que

\section{Les savoir-faire}

Des outils pour fabriquer la nature et l'espace et à la fois des moyens d'action et d'intervention sur le territoire (L'Écho des Écrins. Le journal dinformation du Parc Nationa/14, automne 2000).

(Fig. 6)
Les enjeux du bocage

« Derrière la Haie ». Vidéocassette produite par Aster - Parc National des Écrins

(Film de Xavier Petit).

(Fig. 7)

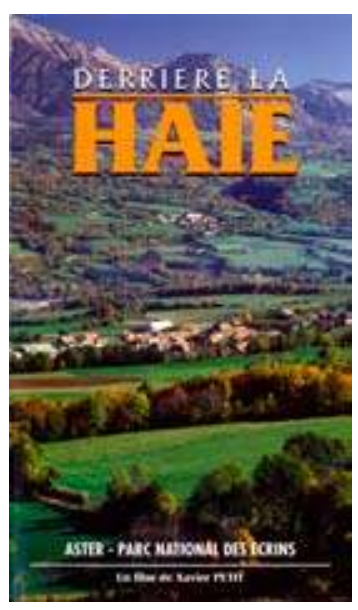




\section{le dauphinè}

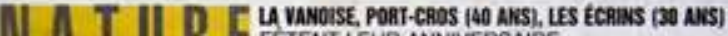 FETENT LEUR ANNIVERSAIRE}
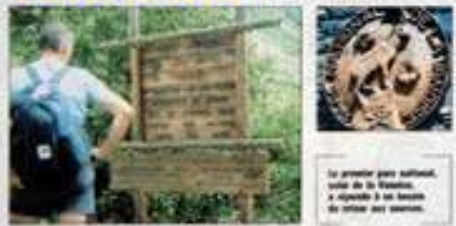

Les parcs nationaux en question

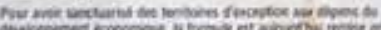

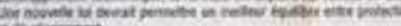

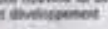

\section{Les 30 ans du Parc National des Écrins}

En attendant le rapport Giran et la nouvelle loi sur les parcs nationaux (Le Dauphiné libéré, 21 juin 2003).

(Fig. 8)

\section{Le nouveau programme d’aménagement du Parc}

La protection de l'environnement śaccompagne de l'aménagement du territoire (L'Écho des Écrins. Le journal d'information du Parc Nationa/9, hiver 1998-99).

(Fig. 9)

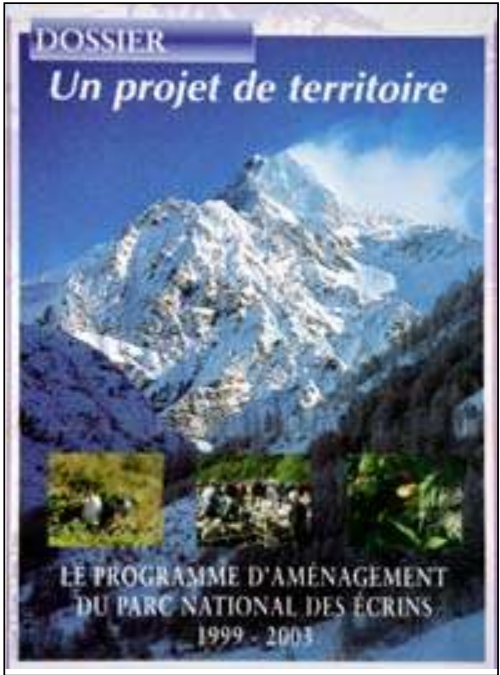

ce parc a en quelque sorte anticipé les changements législatifs au niveau national, qui n'interviendront qu'à partir de 2006.

Au cours de l'été 1996, une «Charte d'environnement et de développement durable ${ }^{7}$, c'est-à-dire un « document cadre de partenariat », a été préparée et signée par les représentants du Parc national des Écrins et par les élus des communes du parc. Dans les documents officiels du parc, tout comme dans les discours de son personnel, l'adhésion à ce document est présentée comme un geste symbolique extrêmement fort, et le signe d'un changement radical dans les interactions entre l'institution et le contexte local. La notion de patrimoine, dans sa double qualification naturelle et culturelle, apparaît comme le fil conducteur de la charte:

« L'enjeu d'un développement durable de ce territoire répond à ces caractéristiques: maintenir et développer la présence et l'activité humaines, en s'appuyant, tout en les préservant, sur les ressources patrimoniales, naturelles et culturelles » $(1996: 1)$

Lencouragement à la valorisation du patrimoine est dicté par la possibilité de l'utiliser à des fins touristiques mais, dans le même temps, celui-ci apparaît de plus en plus connoté comme étant l'élément en mesure de façonner le territoire et, donc, d'en construire l'identité. Le parc, en sa qualité de gestionnaire d'une partie de cet espace, se présente comme l'institution en mesure à la fois d'en décréter la valeur patrimoniale et de fournir une image unificatrice de ce territoire, tout en intégrant les « voix » des différentes vallées:

« Le territoire du Parc national des Écrins présente une richesse et une diversité de sites naturels et culturels qui incitent à marquer la spécificité de cet espace. En effet, cette image de marque constitue le "produit plus", en terme de marketing [...]. Afficher cette identité autour de l'appartenance à un même ensemble, reconnu au plan européen, donnera aux visiteurs une image, un signe de reconnaissance » (Ibid. : 4).

La Charte est ainsi conçue comme un cadre théorique à l'intérieur duquel sont élaborés divers contrats de partenariat entre le parc et des communes ou des ensembles de communes faisant partie d'un même « secteur ». La plus grande partie des contrats a été signée entre 1999 et 2000, alors que je commençais mon travail de recherche dans les Hautes-Alpes. L'un des objectifs principaux de ces contrats est souvent de « valoriser le patrimoine culturel [...] [et] transmettre l'identité du territoire et les savoir-faire locaux ». « L'héritage patrimonial, naturel et culturel » est présenté comme « dense et diversifié » et « la conservation des richesses naturelles et culturelles des sites [comme] indispensable au maintien de la valeur de ce territoire $»^{8}$. Le parc et les collectivités locales, en tant que partenaires, s'engagent à « préserver les milieux, leur diversité et les pratiques humaines associées » 
et à « mettre en valeur les sites à forte valeur patrimoniale, vecteurs de la transmission de la culture locale », parce que « les héritages patrimoniaux et les particularités locales revêtent eux aussi un intérêt économique majeur ${ }^{9}$.

La « culture locale » et les pratiques humaines semblent désormais faire partie des milieux, et les richesses naturelles deviennent indissociables des richesses culturelles.

Par ailleurs, les éléments connotés comme patrimoniaux, qui donnent de la valeur au territoire, ont également une valeur économique. La qualification patrimoniale du territoire crée ainsi un terrain de jeu en apparence neutre et consensuel. Ce qui semble être un accord, n'est en réalité qu'un consensus temporaire, recouvrant des conflits de pouvoir qui eux, en revanche, sont permanents. Derrière une apparence « contractuelle » et de partenariat autour du patrimoine, apparaissent des stratégies et des intérêts divers.

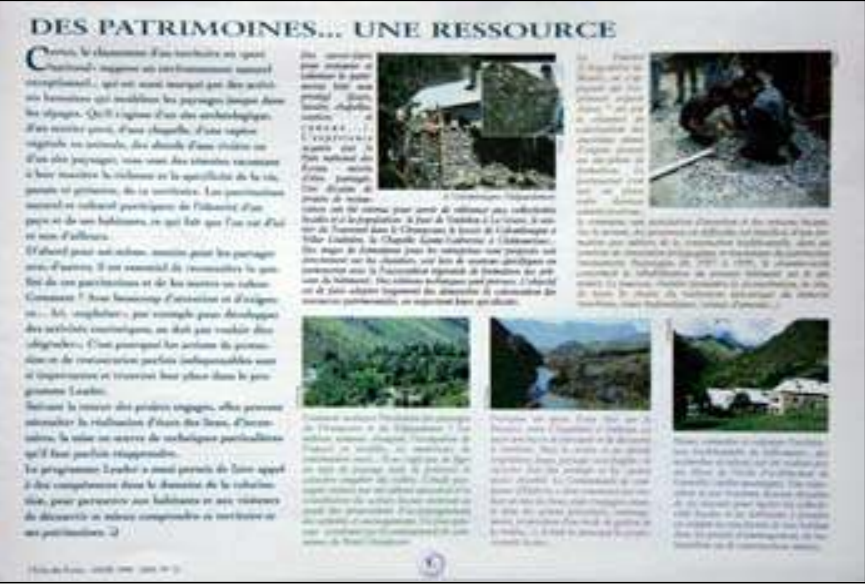

« La charte est un moyen pour que les communes acceptent davantage le parc. Les actions collectives et de partenariat permettent de faire accepter le parc; c'est une sorte d'effet induit. La population locale n'accepte pas le parc et la protection forte ne peut pas être le seul moyen » (T.T. chef de service).

« Nous nous sommes efforcés d'intégrer la dimension du patrimoine culturel parce qu'elle permet le partenariat, et le partenariat est un moyen pour maintenir le patrimoine naturel et culturel [...] (mais) beaucoup de gens disent que ce n'est pas à nous de travailler sur le patrimoine culturel » (L.L. chargé de mission).

Est en jeu non pas la gestion, sur le plan administratif, d'un espace géographique sur lequel les compétences semblent désormais claires, mais sa gestion au niveau symbolique (qui a le droit de « dire » le territoire et d'en définir l'image?), ainsi que sa gestion en termes économiques (non seulement la gestion de ses ressources mais aussi la définition de ses ressources). La notion de patrimoine se transforme ainsi en instrument, pour les uns comme pour les autres, d'une telle négociation. Les représentants des communes, tout comme une partie des élus, semblent l'intégrer dans leurs pratiques:

« Lorsqu'ils ont compris que le patrimoine pouvait constituer une richesse et qu'il pouvait favoriser un tourisme de masse, les communes et le Conseil Général ont commencé à s'intéresser au patrimoine. Ils avaient fait de même avec les stations de ski et à présent, ils adoptent la même stratégie, le même comportement avec le patrimoine. Pour le parc, le patrimoine est un prétexte, un thème fondateur pour faire travailler les gens ensemble » (L.L. chargé de mission).

La notion de «patrimoine culturel », qui avait en quelque sorte permis l'entrée de l'environnement et de la nature dans l'espace patrimonial, réapparaît dans un contexte où, depuis longtemps désormais, 


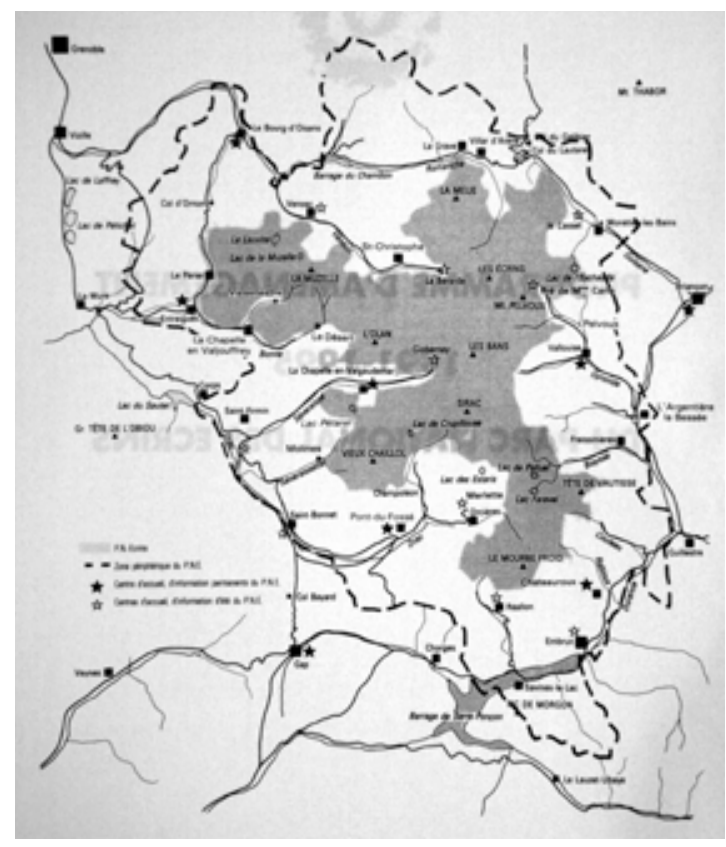

nature et environnement semblaient détachés de la dimension culturelle. La dimension culturelle permet-elle alors de continuer à penser la nature ou plutôt de déplacer les intérêts et donc les enjeux?

En effet, le processus en cours est plus complexe qu'il n'apparaît. Il ne s'agit pas simplement d'un changement au niveau du discours et des pratiques: à travers la « patrimonialisation » d'objets, de savoirs et savoir-faire locaux, le parc et ses agents s'engagent dans le processus de façonnement du territoire, créent des « communautés imaginées » (Anderson 1991) - les vallées des « territoires Écrins » - au sein d'un espace « naturel » protégé, et par là construisent et négocient leur légitimité d'action sur cet espace.

1990, Carte du Parc National des Écrins

(Programme dáménagement du Parc National des Écrins 1991-1995, source Parc national des Écrins).

(Fig. 11)

\section{5, Carte du Parc National des Écrins}

(Rapport d'activité 1995, source Parc national des Écrins).

(Fig. 12)

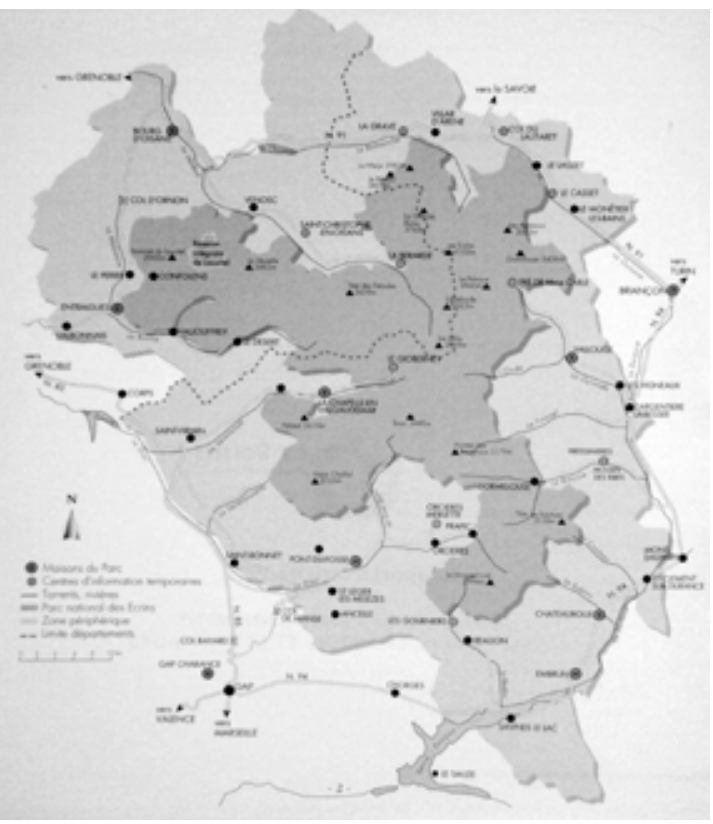

Les questions relatives à la « localité » et à la construction/définition des lieux sont parmi les thèmes qui animent le débat anthropologique en Europe et aux États-Unis. Le sens donné ici à la notion de localité renvoie, entre autres, aux travaux d'Appadurai (1996), de Gupta et Ferguson (1997, 2001), de Low et Lawrence-Zùniga (2003). La « localité » n'est pas un donné. Mais bien au contraire, localité et appartenance ont une nature relationnelle et contextuelle: elles sont constamment remodelées et affirmées à travers des performances qui mettent en scène le « lieu » et la « communauté » (Abram et al. 1997 : 3-4). Les individus ont un besoin constant de définir et d'affirmer la « localité » et leur appartenance, perçues comme fragiles et menacées. La production de la localité (the production of locality, Appadurai 1996: 178) a donc des effets matériels sur les espaces qui sont ainsi transformés en lieux; et, en même temps, elle contribue à construire des sujets locaux et structure les sentiments d'appartenance. Il s'agit des deux aspects de ce qu'Appadurai définit comme une « technologie générale de la localisation », en tant que propriété de la vie sociale. La transformation d'un espace en lieu est toujours liée aux configurations du pouvoir: le processus de « localisation » est ainsi un processus politique (Gupta \& Ferguson 2001 : 8) et un enjeu dans les dynamiques sociales ${ }^{10}$.

Dans ce sens, les espaces protégés peuvent être considérés, à certains égards, comme des « sites contestés». Dans le cadre d'une anthropologie de la localité, Low et LawrenceZúñiga définissent ces derniers comme « des lieux géographiques dans lesquels des conflits sous forme d'opposition, de confrontation, subversion et/ou résistance engagent les acteurs dont les positions sociales sont définies en termes de 
contrôle différentiel des ressources et d'accès au pouvoir » (Low et Lawrence-Zúñiga 2003 : 18). Le pouvoir de ces sites est lié à leur capacité de fonctionner comme des symboles pour communiquer à travers une condensation de significations (ibid. 19). Cette stratification de sens est particulièrement visible dans le Parc national des Écrins et il est possible de lire les processus en cours au sein de son territoire comme des processus de production de la localité.

Les cartes élaborées par le parc sont à la fois un outil et un produit de ces dynamiques. J'ai déjà analysé les tentatives de construction d'une identité commune autour du parc à travers la notion géographique de « massif » et le nom d' « Écrins ». Avant la création du parc, «Écrins » désigne simplement le Col et la Barre des Écrins (Fig. 1) - deux éléments qui marquent la frontière physique entre les deux départements, l'Isère et les Hautes-Alpes. Le terme de « massif » devient par la suite un synonyme du parc (des Écrins) auquel il emprunte son nom, en devenant le « massif des Écrins » (cf. Siniscalchi 2007).

Dans les documents produits au début des années 1990 et jusqu'en 1995, le territoire du parc est représenté par des cartes dans lesquelles la zone centrale est colorée en gris et les limites avec la zone périphérique signalées de manière bien visible; par contre, le périmètre de la zone périphérique est dessiné d'un trait fin qui ne le distingue pas nettement de l'espace extérieur (Fig. 11). Les limites de la zone périphérique deviennent quant à elles de plus en plus visibles dans les documents de la fin des années 1990 (Fig. 12). Ces années sont celles de l'élaboration et de la réalisation d'un projet « Leader II », qui associe des communes ne faisant pas partie

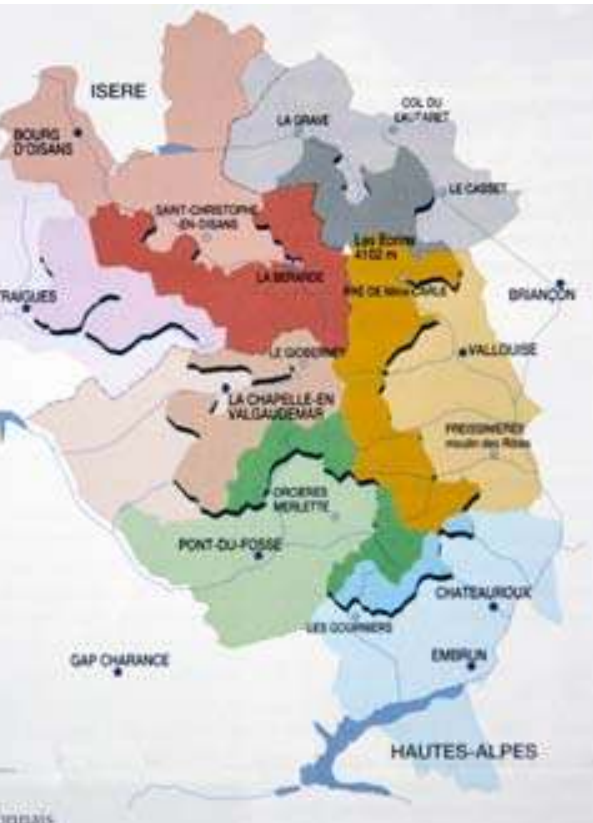
du parc mais limitrophes: le but affiché est de maintenir la cohérence des actions envisagées. À cette époque, le territoire du parc commence à être représenté comme un espace plus large dans lequel les limites entre zone périphérique et zone centrale tendent à disparaître. Les unités pertinentes - souvent en couleur - sont plutôt les vallées qui forment la structure du massif des Écrins (Fig. 13). Autour de ces traits en évolution se joue la légitimité d'action du parc et de son personnel sur un espace plus vaste que celui auquel s'applique le décret de protection. Enfin, le document de présentation du projet « Leader $+{ }^{11}$ présente le parc à travers une carte dans laquelle les limites entre les deux zones sont devenues désormais invisibles, tout comme celles entre les communes du parc et les autres, qui sont simplement des communes associées au projet (Fig. 14). Les données auxquelles font référence ces cartes, ne sont pas des entités stables et immuables. Il s'agit au contraire d'instruments flexibles, utilisés dans les jeux de pouvoir que j'ai essayé de retracer et d'analyser ici.

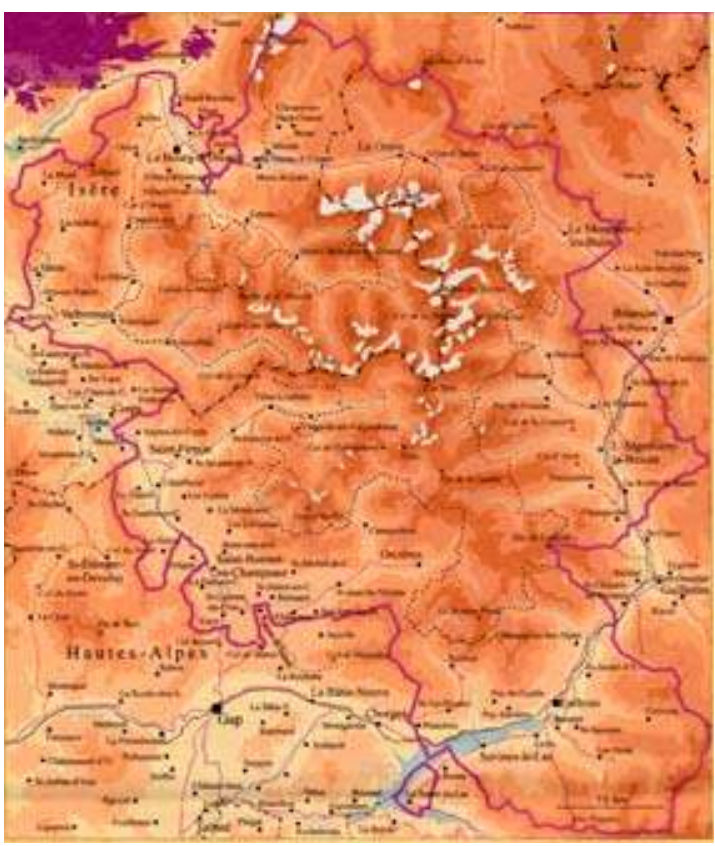


Du point de vue du parc, l'attention particulière portée au patrimoine, et plus précisément au patrimoine culturel - également visible dans ces projets -, permet de représenter le parc non plus comme l'institution protectrice de la nature (même si ce rôle est constamment sous-entendu), mais comme un partenaire dans le développement du contexte local. En 2001, un nouveau directeur est nommé; il s'agit de l'ancien directeur du parc naturel régional du massif des Bauges. « les parcs naturels régionaux ont été les premiers à intégrer le regard naturaliste avec un regard sur la culture. Ce qui les relie c'est l'homme » affirme-t-il, soulignant ainsi la continuité entre son ancienne fonction et l'actuelle.

Lattention au patrimoine - désormais de plus en plus indiqué au pluriel dans les documents administratifs, «les patrimoines »-devient ainsi un outil de gestion des ressources économiques et un instrument de légitimation politique. Elle est à la fois l'un des instruments et l'un des résultats d'un processus plus large de production de la localité.

Ce qui semblait être une dichotomie en termes d'intérêts (la nature contre l'économie) ou une convergence (le patrimoine pour l'économie) sont des manières d'exprimer ou d'occulter des rapports de force et des jeux de pouvoir. À travers la nature et l'environnement, transformés en objets patrimoniaux et maintenant en biens culturels, les acteurs sociaux peuvent construire et manipuler différents niveaux d'identité, et lutter pour la gestion des ressources et la définition des actions économiquement efficaces. Ils construisent et reconstruisent ainsi l'espace local. 


\section{NOTES}

1. D'autres aspects des processus analysés dans cet article ont été publiés ailleurs, cf. Siniscalchi 2002b, 2007.

Je tiens à remercier tout particulièrement les responsables et les personnels du Parc pour leur disponibilité et leur aide précieuse tout au long de mon travail. Une partie de cette recherche a été possible grâce à une bourse de la Fondation Fyssen.

2. La Loi 2006-436 du 14 avril 2006 et son décret d'application reposent différemment le problème des limites d'un parc national à travers l'idée de « zone optimale d'adhésion », « définie comme tout ou partie du territoire des communes qui, ayant vocation à faire partie du parc national en raison notamment de leur continuité géographique ou de leur solidarité écologique avec le cœur, ont décidé d'adhérer à la charte du parc national et de concourir volontairement à cette protection » (article 1).

3. Par R. Brichet, administrateur civil qui travaillait à la sous-direction des Monuments et des Sites.

4. Programme d'aménagement 1991-1995. 1990, Gap, Parc National des Ecrins.

5. En 1994, le rapport ministériel Une politique pour le patrimoine culturel rural - connu comme « Rapport Chiva »-participe à la définition et à la construction de la notion de « patrimoine culturel rural », en mettant l'accent sur la nécessité de passer de formes de protection contraignantes à des nouvelles modalités de gestion des territoires : « il faut se doter des instruments d'une meilleure intégration du patrimoine culturel dans les politiques globales d'aménagement du territoire » (1994:99). Ce rapport prêtait une attention particulière à la dimension économique de la gestion du patrimoine.

6. Les Parcs Nationaux et l'Aménagement du Territoire, 19 mai 1994. Document édité par les Parcs Nationaux de France: 2-4.

7. Charte d'environnement et de développement durable. Document cadre de partenariat entre le Parc national des Écrins et les communes du Parc, Gap, Parc National des Écrins, juillet 1996.

8. Contrat de partenariat, Charte d'environnement et de développement durable, Secteur du Briançonnais, 2 juillet 1999, Parc National des Écrins.

9. Contrat de partenariat, Charte d'environnement et de développement durable, Secteur de l'Embrunais, 10 juillet 2000, Parc National des Écrins.

10. Cf. Anderson 1991. Sur les conflits dans la construction de l'identité des lieux, et plus en général de la localité cf. entre autres Abram, Waldren, Macleod 1997, Appadurai 1996, Goddard, Llobrera, Shore 1994, MacDonald 1993; cf. aussi, pour certains aspects, Siniscalchi 2002a. Pour les Hautes-Alpes, Siniscalchi 2003.

11. Dans les Écrins. Dossier de candidature au Programme d'Initiative Européenne Leader +. Août 2001, Gap, Parc National des Écrins. 


\section{RÉFÉRENCES}

Abram, Simone, Waldren, Jacqueline \& Macleod, Donald, V. L., (eds), 1997, Tourists and Tourism. Identifying with People and Places. Oxford-New York, Berg.

Anderson, Benedict, 1991, Imagined Communities. Londres-New York, Verso.

Appadurai, Arjun, 1996, Modernity at Large. Cultural Dimensions of Globalisation. Minneapolis, University of Minnesota Press.

Audrerie, Dominique, 1997, La Notion et la protection du patrimoine. Paris, PUF.

Avocat, Charles, 1977, Le Parc National des Écrins. Gap, Société d'études des Hautes-Alpes.

Babelon, Jean-Pierre, Chastel, André, 1994, La Notion de patrimoine. Paris, Liana Levi.

Certeau, Michel de, 1990, L'Invention du quotidien, I, Arts de faire. Paris, Gallimard.

Chastel, André, 1997, « La notion de patrimoine », in Pierre Nora, (ed.), Les Lieux de mémoire, vol I. Paris, Gallimard : 1433-1469.

Chiva, Isac, 1994, Une politique pour le patrimoine culturel rural. Paris, Rapport remis au Ministre de la Culture et de la Francophonie.

Descola, Philippe, 2000, «Lanthropologie et la question de la nature », in M. Abélès, L. Charles, H.-P. Jeudy \& B. Kalaora, (eds), L'Environnement en perspective. Contextes et représentations de l'environnement. Paris, L'Harmattan : 61-84.

Desvallées, André, 1998, «À l'origine du mot "patrimoine" », in D. Poulot, (ed.), Patrimoine et modernité. Paris, L'Harmattan: 89-105.

Donnan, Hastings \& Wilson, Thomas M., 2003, « Territoriality, anthropology, and the interstitial: subversion and support in European borderlands », Focaal. European Journal of Anthropology 41 : 9-20.

Gupta, Akhil \& Ferguson, James, (eds), 1997, Anthropological Locations. Boundaries and Grounds of a Field Science. Berkeley-Londres, University of California Press.

Gupta, Akhil \& Ferguson, James, (eds), 2001, Culture, Power, Place. Explorations in Critical Anthropology. Londres, Duke University Press.

Herzfeld, Michael, 1997, Cultural Intimacy. Social Poetics in the Nation-State. New York, Routledge.

Hirsch, Eric, O'Hanlon, Michael, (eds), 1995, The Anthropology of Landscape. Perspectives on Place and Space. Oxford, Oxford University Press.

Kalaora, Bernard, 1997, «Quand l'environnement devient affaire d'État », in M. Abélès et H.-P. Jeudy, (eds), Anthropologie du politique. Paris, A. Colin: 179-196.

Lascoumes, Pierre, 1994, L'Éco-pouvoir. Environnement et politiques. Paris, La Découverte.

Low, Setha M., Lawrence-Zúñiga, Denise, (eds), 2003, The Anthropology of Space and Place. Locating Culture. Oxford, Blackwell.

Mac Donald, Sharon, (ed.),1993, Inside European Identities. Oxford, Berg.

Siniscalchi, Valeria, 2002a, « Introduzione. Economie al caleidoscopio», in Siniscalchi V., (ed.), Frammenti di economie. Ricerche di antropologia economica in Italia. Cosenza, Luigi Pellegrini Editore: 7-40.

Siniscalchi, Valeria, 2002b, «"Patrimoine naturel" et "patrimoine culturel" dans le Parc national des Écrins (France) : nouvelles représentations des territoires "protégés" », Europaea VIII : 303-317.

Siniscalchi, Valeria, 2003, «Entre Provence et Dauphiné: frontière et identité dans les Hautes-Alpes », Ethnologie française XXIII (1): 101-109.

Siniscalchi, Valeria, 2007, «Le Parc national des Écrins et la construction de la localité. Usages et représentations du territoire et de la nature dans un espace "protégé" », Cahiers d'anthropologie sociale 3 : 31-46

Szarka, Joseph, 2002, The Shaping of Environmental Policy in France. New York, Oxford, Berghahn Books.

Zuanon, Jean-Paul, 1994, Chronique d'un "parc oublié". Du parc de La Bérarde (1913) au Parc national des Écrins (1973). Grenoble, Revue de géographie alpine. 


\section{RÉSUMÉ}

Économie et pouvoir au sein du parc national des Écrins. Les espaces protégés sont des objets d'observation privilégiés, au sein desquels des logiques d'utilisation du territoire et des intérêts divergents s'expriment et les conflits se révèlent. En suivant les transformations des actions et des discours du Parc national des Écrins au fil de ces dernières décennies, cet article s'intéresse tout particulièrement aux politiques et aux rhétoriques à travers lesquelles cette institution est devenue un acteur du processus de construction de l'espace. Dans les premières années de vie du parc (années 1970), la « nature » apparaît comme étant au centre de ses préoccupations. Le passage de l'idée d'une nature à préserver à la notion de « patrimoine naturel » d'abord, et « culturel » ensuite, accompagne les transformations qui se sont produites dans les logiques et dans les formes de protection de la nature des années 1960 à nos jours. En même temps, il est le signe de changements, radicaux à certains égards, dans les rapports entre le parc et le contexte local.

Comment l'idée de nature se modifie-t-elle au sein de ce processus? Quel sens prend-elle aujourd'hui? Aux transformations des notions utilisées correspondent des changements de posture et d'actions, qui sont à leur tour révélateurs des changements survenus au niveau des enjeux (politiques et économiques). La vision patrimoniale de l'espace semble favoriser une prise en compte simultanée des aspects naturels et humains. Alors qu'au moment de sa création le parc était perçu au niveau local comme un obstacle au développement économique, la nouvelle vision patrimoniale lui permet de se présenter comme l'un des acteurs de ce développement et de réintroduire la dimension économique.

À travers la nature, l'environnement, les savoirs et les savoir-faire, tous connotés en termes culturels et patrimoniaux, les acteurs sociaux construisent différents niveaux d'identité et modèlent le territoire. Les politiques de la localité que le parc met en œuvre à travers ses actions et ses discours révèlent des jeux de pouvoir dans lesquels le parc est un acteur local parmi d'autres, tout en étant l'expression du pouvoir de l'État.

\section{ABSTRACT}

Economy and Power within the Parc National des Ecrins. Protected spaces are privileged objects for observing uses of territory, their logics as well as the divergent interests and conflicts around them. This article looks at changing actions and discourses in the Parc National des Ecrins over the last few decades, in order to examine the policies and rhetorics that have transformed this institution into an actor in the construction of space. In the first years of the park's existence (1970s), "nature" appears to have been at the centre of its preoccupations. The shift from the idea of a nature that had to be preserved to the notion first of a "natural" and then of a "cultural heritage" accompanied the transformations of logics and forms of protection of nature from the 1960s until today. At the same time, it indicates the sometimes radical changes in the relations between the park and the local context.

How is the idea of nature modified within this process? What does it mean today? Changes in notions correspond to changes in postures and actions, which in their turn reveal changed stakes (political and economic). The vision of space as heritage seems to favour the simultaneous consideration of natural and human aspects. While at the local level, the park was perceived at its creation as an obstacle to economic development, the new vision of heritage allowed it to present itself as one of the actors of this development and reintroduce the economic dimension.

Social actors construct different levels of identity and produce their space and place through nature, environment, knowledge and skills, transformed into heritage. This politics of locality shows the power competitions in which the park is one of the actors.

\section{MOTS CLÉS}

Parc, espace, pouvoir, nature, patrimoine, anthropologie de l'espace, lieu.

\section{KEYWORDS}

Park, space, place, power, nature, heritage, anthropology of space. 